\title{
Synthesis
}

\section{Three-Way Interactions among Mutualistic Mycorrhizal Fungi, Plants, and Plant Enemies: Hypotheses and Synthesis}

\author{
Alison E. Bennett, ${ }^{*}$ Janice Alers-Garcia, ${ }^{\dagger}$ and James D. Bever ${ }^{\ddagger}$
}

Department of Biology, Indiana University, Bloomington, Indiana 47405

Submitted May 9, 2005; Accepted September 13, 2005; Electronically published December 19, 2005

Online enhancement: appendix.

\begin{abstract}
A number of studies have shown that an association with mycorrhizal fungi can alter the outcome of interactions between plants and their enemies. While the directions of these effects vary, their strength suggests the need for greater attention to multispecies interactions among plant enemies, plants, and mycorrhizal fungi. We recognize that mycorrhizal fungi could effect plant enemies by improving plant nutrition, modifying plant tolerance, or modifying plant defenses. In addition, mycorrhizal fungi could directly interfere with pathogen infection, herbivory, or parasitism by occupying root space. We formalize these alternative outcomes of multispecies interactions and explore the long-term dynamics of the plant-enemy interactions based on these different scenarios using a general model of interactions between plants and plant enemies. We then review the literature in terms of the assumptions of the alternative mechanisms and the predictions of these models. Through this effort, we identify new directions in the study of tritrophic interactions between enemies, plants, and soil mutualists.
\end{abstract}

Keywords: mycorrhizal fungi, multitrophic interactions, plant defense, tolerance, plant enemy, mathematical model.

Species interacting within communities form a complex web of associations. As ecologists, we often reduce this complexity by studying pairwise interactions between species. For example, extensive work has focused on inter-

\footnotetext{
* Corresponding author. Present address: Department of Evolution and Ecology, 4348 Storer Hall, University of California, Davis, California 95616; email: alibenne@bio.indiana.edu.

† E-mail: jalersga@bio.indiana.edu.

‡ E-mail: jbever@bio.indiana.edu.
}

Am. Nat. 2006. Vol. 167, pp. 141-152. (C) 2006 by The University of Chicago. 0003-0147/2006/16702-41066\$15.00. All rights reserved. actions between plants and herbivores, while a separate, large body of work exists on the interactions between plants and mutualistic mycorrhizal fungi. It is possible that the dynamics of a more complicated community involving both antagonistic and mutualistic interactions could be resolved by combining the results from studies of pairwise interactions. However, if pairwise interactions are modified by a third species, then a simple combination approach will not accurately portray the dynamics of more complex communities (Wootton 1994; McPeek and Miller 1996; Miller and Travis 1996). Recently, a number of studies have shown that an association with mycorrhizal fungi can change the outcome of interactions between plants and their enemies (reviewed in Borowicz 2001; Gange and Brown 2002; Gehring and Whitham 2002). Results have varied, suggesting that mycorrhizal fungi can alternately benefit the plant or the plant enemy. In addition, mycorrhizal fungi could directly interfere with infection by pathogens, with herbivory, or with parasitism by nematodes and other plants, either chemically or by occupying root space. Here, we delineate potential mechanisms through which mycorrhizal fungi may affect plant enemies, and we explore implications of these different scenarios for the long-term dynamics of the plant-enemy interactions.

\section{Pairwise Plant-Plant Enemy Interactions}

Numerous enemies besiege plants of every habitat and growth form. Within the class Insecta alone, an estimated 361,000 species feed on a wide variety of plants (Strong et al. 1984); bacterial and fungal pathogens attack both plant shoots and roots; and plant, nematode, and insect parasites suck nutrients out of their host plants. Plants have developed a wide array of mechanisms for defending against plant enemies, and these defenses can be grouped into three categories: tolerance, constitutive resistance, and induced resistance. Plants can exhibit all three strategies at various stages of enemy attack. Tolerance refers to a plant's ability to vegetatively or reproductively overcome damage by herbivores (Strauss and Agrawal 1999). Some 
plants may be able to overcompensate for damage by producing a larger biomass or greater number of seeds (Paige and Whitham 1987). We define constitutive resistance as any mechanism the plant employs before any herbivore attack occurs that results in reduced damage by herbivores. Induced resistance is the ability of a plant to developmentally change after receiving herbivore damage that results in reduced future damage by herbivores (Karban and Baldwin 1997). For example, plants may produce new thorns or thicker leaves (Kudo 1996) or upregulate the production of defensive chemicals (Baldwin 1994).

\section{Pairwise Plant-Mycorrhizal Fungi Interactions}

Mycorrhizal fungi form symbiotic associations with plants that can often be mutually beneficial to both plant and fungus. Association with mycorrhizal fungi can increase plant access to scarce or immobile soil minerals, particularly phosphorus (for arbuscular mycorrhizal [AM] fungi) and nitrogen (for ectomycorrhizal and ericoid mycorrhizal fungi), thereby increasing plant growth rates (Smith and Read 1997). In return, the fungus derives dependent carbohydrates from the plant—some fungi cannot grow without a suitable host plant. Plant-mycorrhizal fungi interactions span a continuum from mutualism to parasitism, however. For example, in environments high in soil nutrients, the fungus may parasitize the plant (Johnson et al. 1997). In addition, numerous studies have shown that individual species of AM fungi differ in their ability to promote plant growth and that growth enhancement can depend on the particular matching of plant and fungal species (Van der Heijden et al. 1998; Bever 2002; Klironomos 2003). Here, we primarily focus on mycorrhizal associates that provide a measurable benefit to their host.

We develop a novel framework for classifying interactions among plants, plant enemies, and mycorrhizal fungi. Numerous articles have documented mycorrhizal mediated plant responses to soil pathogens (Borowicz 2001) and belowground herbivores (Gange and Brown 2002). However, fewer articles have focused on aboveground herbivores (Gehring and Whitham 2002), and very few articles have addressed aboveground parasites (Sanders et al. 1993) or other kinds of plant enemies. Prior research suggests several possible outcomes of the three-way interaction among plants, plant enemies, and mycorrhizal fungi, and these outcomes may be generated by different mechanisms. For example, Gange and his collaborators have shown in Plantago lanceolata that fungal colonization reduces herbivore damage (Gange and West 1994). On the other hand, Gehring and Whitham's group has shown that despite a negative effect of herbivory by scale insects on ectomycorrhizal colonization, there was no effect of ectomycorrhizal colonization on the level of plant damage or scale insect mortality (although scale insect growth rate was not measured; Gehring et al. 1997). Other work has demonstrated the potential for mycorrhizal fungi to directly interfere with attack by root pathogens (Newsham et al. 1995; Borowicz 2001).

Specifically, we address the following questions. First, through what mechanisms may mycorrhizal fungi alter the outcomes of interactions between plants and plant enemies? We explore both direct (e.g., occupation of root space) and indirect (e.g., changes in plant nutritional value, tolerance, or defense) pathways, and we review the current literature in the context of this framework. Second, how do mycorrhizal fungi influence the long-term dynamics of plant-plant enemy interactions? We use simple modifications of the Lotka-Volterra predator-prey equations to identify qualitatively different dynamical implications of each mechanism.

\section{Potential Mechanisms}

We begin by assuming that plants derive nutritional benefit from an association with mycorrhizal fungi. We then differentiate one direct and four indirect pathways through which the improvement in plant nutrition could alter the interaction between plants and their enemies.

\section{Increase in Available Plant Quantity}

It is possible that through improved plant nutrition, mycorrhizal fungi increase the quantity of the plant enemy's food with no change in plant quality. A change in food quantity could occur through increased growth or increased plant nutrient status, and we refer to this change as the "nutritional quantity hypothesis" and depict it in figure $1 a$. We would expect to see the nutritional quantity hypothesis acting in cases where no indirect interactions occur, and simply combining the outcomes of pairwise interactions predicts the outcome of multitrophic interactions.

\section{Increase in Plant Nutritive Value}

Alternatively, an increase in food quality could occur through changes in plant nutritional content (as proposed by Gange 2005) or a decrease in the concentration of plant defenses in plant tissues, and we refer to this change as the "nutritional food quality hypothesis." This increase in food quantity or quality may translate directly to an increase in enemy performance, and it is depicted in figure $1 b$. 

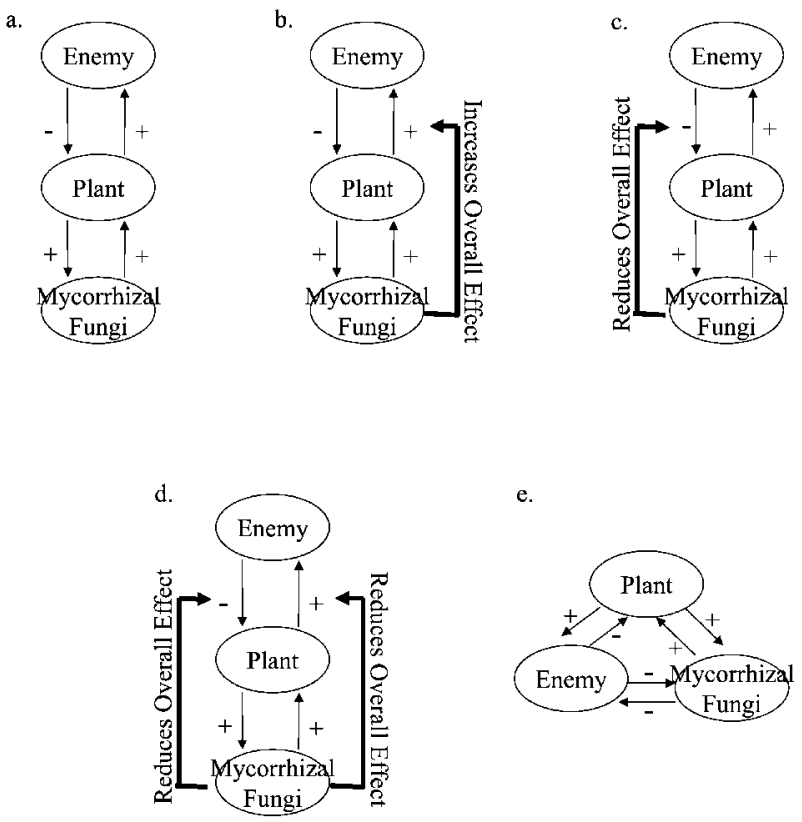

e.

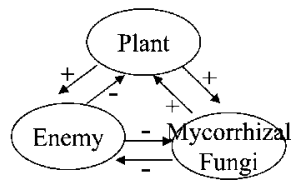

Figure 1: Representations of the five ways in which mycorrhizal fungi could alter interactions between plants and their enemies. Each organism in the multilevel interactions described is represented by a circle, and the arrows in the diagrams represent the flow of energy or resources between the organisms. The sign next to the arrow represents the benefit of the interaction. For example, a plus sign represents a benefit conferred to the organisms. Additional arrows represent indirect modifications of the initial interaction. $a$, Nutritional quantity hypothesis. Arbuscular mycorrhizal fungi improve plant growth, leading to an advantage for the enemy. There are no indirect interactions occurring in this scenario. $b$, Nutritional quality hypothesis. Arbuscular mycorrhizal fungi improve plant nutritional quality, leading to an advantage for the enemy. In this case, mycorrhizal fungi indirectly alter the relationship between the plant and the enemy, as indicated by the additional arrow. $c$, Modification of tolerance hypothesis. Mycorrhizal fungi increase the tolerance of the plant to the enemy, thereby increasing plant fitness. In this case, mycorrhizal fungi indirectly alter the relationship between the plant and the enemy, as indicated by the additional arrow. $d$, Modification of defense hypothesis. A disproportionate change in chemical or physical defenses occurs due to the association between the plant and arbuscular mycorrhizal fungi, leading to decreased enemy fitness. In this case, mycorrhizal fungi indirectly alter the relationship between the plant and the enemy, as indicated by the additional arrows. $e$, Interference hypothesis: arbuscular mycorrhizal fungi directly interfere with the ability of an enemy to attack a plant. There are no indirect interactions occurring in this scenario because mycorrhizal fungi are directly interacting with the plant enemy.

\section{Increase in Plant Tolerance}

Alternatively, under the "modification of tolerance hypothesis," a plant associating with mycorrhizal fungi experiences increased tolerance (fig. 1c). In this scenario, although mycorrhizal fungi may or may not contribute to plant growth in the absence of plant enemies, they do contribute to plant growth following damage by plant en- emies. Such a response would occur if mycorrhizal fungi indirectly alter plant storage patterns, thereby allowing plants to overcome tissue loss after enemy attack (Borowicz 1997; Kula et al. 2005). One can imagine that an increase in plant tolerance might effect herbivores with varying life cycles differently. If the plant responds to damage by regrowth of lost vegetative tissue, then herbivores would benefit in the short term through the replacement of lost tissue. However, if the plant responds to damage by growth of reproductive tissue, then herbivore offspring are more likely to benefit.

\section{Increase in Plant Defense}

It is also possible that the improvement in plant nutrition allows plants to disproportionately increase allocation to enemy defense, that is, to increase defense per unit of plant tissue (Rabin and Pacovsky 1985; Gehring et al. 1997; Wurst et al. 2004). Under the "modification of defense hypothesis," association with mycorrhizal fungi could decrease the performance of plant enemies (fig. 1d).

\section{Interference as Defense}

The effect of mycorrhizal fungi on plant enemies may not be mediated through plant physiology but rather may result from direct interactions between mycorrhizal fungi and plant enemies. Researchers in agriculture have long known that mycorrhizal fungi decrease damage by root pathogens and nematodes (reviewed in Borowicz 2001). In the interference hypothesis (fig. 1e), mycorrhizal fungi directly interfere with plant enemies by inhibiting enemies from attacking the host. This interference is most likely to occur in plant roots, where mycorrhizal fungi may directly interfere chemically with plant enemies or occupy "root space" that might otherwise be occupied by plant enemies such as root pathogens or nematodes.

These four hypotheses represent extreme possibilities but are not mutually exclusive because plants can respond through multiple pathways to a single interaction with mycorrhizal fungi. However, it is possible that trade-offs exist in a plant's allocation of resources among growth, tolerance, and defense such that a plant's ability to allocate more resources to one of the three strategies means that resources cannot be allocated to other strategies. We can represent this trade-off as a plane positioned in three dimensions that constrains possible phenotypes (fig. 2). This type of trade-off has been an implicit assumption in many of the discussions of plant defense theories (such as the optimal defense hypothesis [Rhoades 1979], the growth rate hypothesis [Coley et al. 1985], and the growthdifferentiation balance hypothesis [Loomis 1932; Loomis 1953]) and has been empirically supported in several stud- 


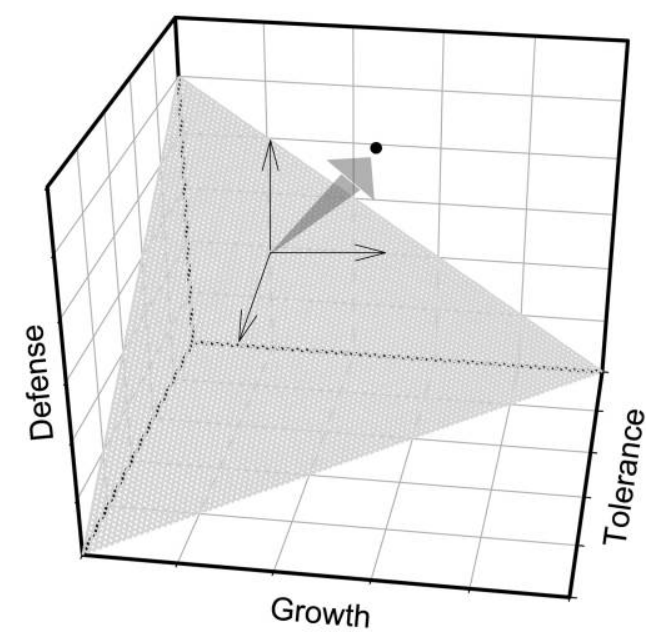

Figure 2: Trade-off between growth, tolerance, and defense. Growth, tolerance, and defense are graphed as three axes. Without mycorrhizae, movement toward one of the three variables causes a reduction in the other two variables such that plant phenotypes are constrained to the graphed trade-off plane. However, mycorrhizae allow movement off the trade-off plane such that a plant can increase allocation in multiple dimensions at once.

ies (Fineblum and Rausher 1995; Stowe 1998; Fornoni et al. 2003; Prittinen et al. 2003; Strauss et al. 2003; but see Mole 1994; Karban and Baldwin 1997). Plants associated with mycorrhizal fungi can use the additional resources to step off this trade-off plane described within many plant defense hypotheses and increase allocation to one or more axes (growth, defense, or tolerance) without decreasing allocation to any of the other axes (fig. 2).

Different plant species are likely to respond differently to mycorrhizal infection as well as plant damage, and a number of plant-defense hypotheses provide guidance for understanding how this might occur. The two most applicable hypotheses are the "carbon-nutrient balance hypothesis" and the "growth-differentiation balance hypothesis." Both of these hypotheses suggest that plant allocation to defense, or other non-growth-related functions, is dependent on the ratio of nutrients (particularly nitrogen) to carbon available for plants. In particular, whenever nutrients are limiting, plants are likely to allocate resources to defense or non-growth-related differentiation. The growth-differentiation balance hypothesis also accounts for the possibility that nutrient limitations may also limit photosynthesis, and such a case leads to a moderate level of differentiation and a reduction in the growth rate. Arbuscular mycorrhizal fungi act as a carbon and nitrogen sink for plants (Smith and Read 1997; Miller et al. 2002); however, they also increase plant photosynthetic rate while increasing plant uptake of nutrients. As a result, they can influence the carbon-to-nutrient ratio in a variety of ways. For example, if increased plant photosynthetic rate compensates for the carbon sink created by mycorrhizae, then mycorrhizae are likely to alter the carbon-to-nutrient ratio in favor of carbon and thus in favor of growth. However, if plant photosynthetic rate does not compensate for the carbon sink created by mycorrhizae, the carbon-to-nutrient ratio would shift in favor of nutrients leading to an increase in differentiation. Similar scenarios could be imagined for fluctuations in nitrogen within plants associated with mycorrhizal fungi as well. Therefore, we might expect that, depending on their allocation to mycorrhizal fungi, different plant species are likely to vary in growth, resistance, and tolerance. While it is possible for simultaneous movement in multiple directions along the tradeoff plane due to an association with mycorrhizal fungi, we discuss the three axes (growth, tolerance, and defense) separately in order to fully explore the consequences of the hypotheses outlined above.

\section{Mycorrhizal Modification of Plant-Enemy Dynamics}

\section{A Simple Model}

The different ways in which mycorrhizal fungi can alter interactions between plants and plant enemies will have distinct consequences for the long-term dynamics of the plant-plant enemy interaction. We illustrate these consequences by developing a framework for the interaction between plants and plant enemies analogous to the LotkaVolterra equations used to predict consumer-producer dynamics (Rosenzweig and MacArthur 1963; Harrison 1995). This general approach has been useful for describing general features of plant-herbivore dynamics (Rosenzweig and MacArthur 1963; Caughley 1976; Crawley 1983; Yodzis 1989; Harrison 1995; van de Koppel et al. 1996; Vos et al. 2004). In particular, we assume that increases in plant biomass are limited by the total amount of biomass that can be produced (logistic growth with a carrying capacity, $K$ ) and by plant consumption by a plant enemy. We also assume that enemy consumption of plants is limited by the time needed to find, consume, and digest a plant, generating a saturating (Type II) functional response. Plant enemy growth rates are assumed to be only limited by the availability of plant biomass.

With these major assumptions, we can represent the instantaneous rate of change in plant biomass $d P / d t$ as a function of plant biomass $(P)$ and plant enemy population size $(E)$ with the following equation:

$$
\frac{d P}{d t}=r P\left(1-\frac{P}{K}\right)-(1-g)\left(\frac{f P}{B+P}\right) E
$$


where $r$ is defined as the intrinsic rate of biomass production of the plant, $K$ is the maximum sustainable plant biomass in the absence of the enemy, $f$ is the maximum feeding rate of the enemy on the plant, $B$ is the "half saturation constant" or the biomass of the plant at which the enemy feeding rate is half-maximal, and $g$ is the relative tolerance of the plant to enemy attack. The first half of equation (1) represents biomass production of the plant, and the second half represents biomass loss to the enemy. The expression $(f P / B+P)$ in the second term represents the saturating functional response of the enemy.

Population growth of the enemy $(d E / d t)$ is directly dependent on the biomass of the plant as represented by

$$
\frac{d E}{d t}=\frac{C f P E}{B+P}-d E
$$

where $C$ represents the "conversion efficiency" or the rate at which the enemy converts plant biomass into enemy offspring and $d$ represents the per capita enemy death rate. The first term represents the birth rate of the enemy, which is a function of the plant biomass consumption, and the second term represents the death rate of the enemy, which is assumed to be density independent.

With the formalization of the plant-enemy interaction in equations (1) and (2), we can infer long-term dynamics (Edelstein-Keshet 1988; Yodzis 1989). The long-term dynamics of this model can be visually represented in a phase-plane diagram in which the direction of change over time is represented as arrows (fig. 3). The lines of zero growth can be derived by setting equations (1) and (2), respectively, to 0 and solving for the relationship between the two state variables $E$ and $P$. The isocline of equation (1) is a parabola of the equation

$$
E=\frac{r\left(1-\frac{P}{K}\right)(B+P)}{(1-g) f} .
$$

Thus, initially, the rate of biomass production increases until it reaches a maximal rate (the peak of the isocline defined as $K-B / 2$ ) after which the rate of biomass production decreases to $K$. The isocline of equation (2) is a straight line of the equation:

$$
\hat{P}=\frac{d B}{C f-d}
$$

Thus, the intersection of these two lines $(\hat{E})$, found by substituting $\hat{P}$ for $P$ in the plant isocline, is given by the following equation:

$$
\hat{E}=\frac{r\left(B+\frac{d B}{C f-d}\right)\left[1+\frac{d B}{K(d-C f)}\right]}{f(1-g)} .
$$

Plant consumption by an enemy can reduce the equilibrium plant biomass below its maximum $(K)$, when the

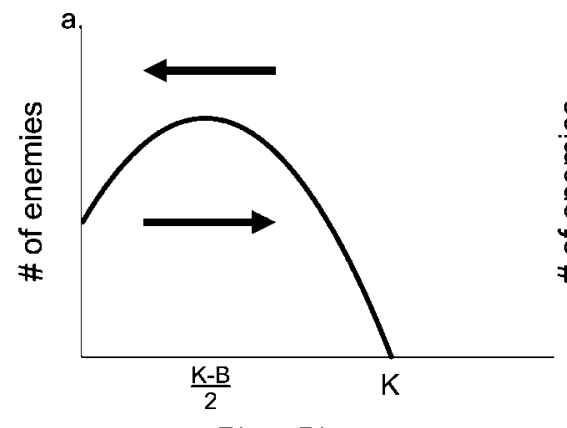

Plant Biomass
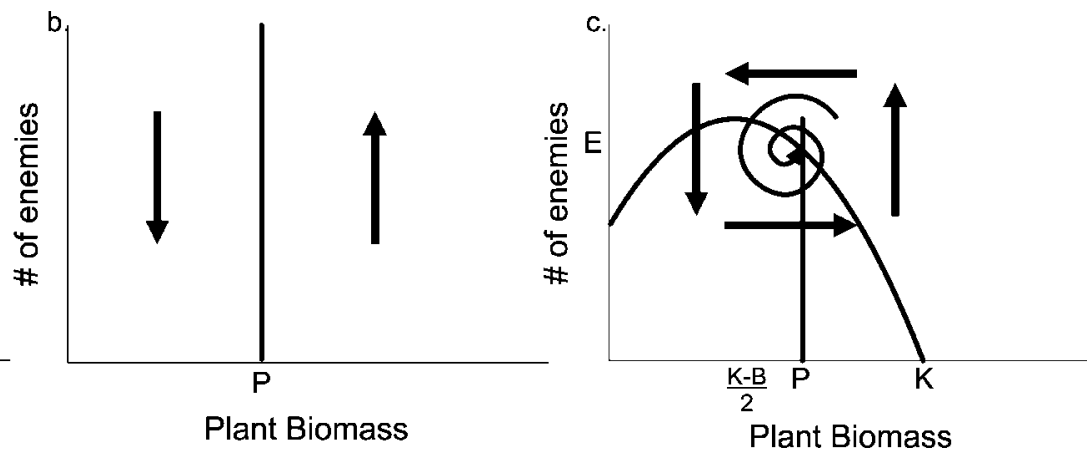

Figure 3: To understand how mycorrhizal fungi might alter the long-term dynamics of plant-herbivore interactions, we modified the Lotka-Volterra predator-prey equations to reflect plant-herbivore dynamics. We then graphed the isoclines of these equations, and they are illustrated. In $a$, we see the plant isocline, a parabola of equation (3) in which $K=200, B=40, C=0.05, t=0$, and $f=0.5$. Below the plant isocline, plant biomass increases, as indicated by the arrow, and above the plant isocline, plant biomass decreases, as indicated by the arrow. In $b$, we see the enemy isocline, a straight line of equation (4) in which $d=0.02, B=40, C=0.05$, and $f=0.5$. To the left of the enemy isocline, enemy numbers decrease, and to the right of the line, enemy numbers increase, as indicated by the arrows. In $c$, we see the plant and enemy isoclines on the same graph. Probability suggests that the enemy isocline is more likely to intersect the plant isocline on the right half of the peak $([K-B] / 2)$, and thus we begin in this configuration. The combination of plant and herbivore isoclines results in dampening population cycles (as suggested by the dampening spiral) of both plant enemy and plants leading to an equilibrial plant biomass production of $\hat{P}$ and an equilibrial enemy population size of $\hat{E}$, indicated by the intersection of the two isoclines. The straight arrows are the combination of arrows from the plant isocline $(a)$ and the enemy isocline $(b)$. 
enemy zero-growth line $(\hat{P})$ is less than maximum production of plant biomass $(K)$. If $\hat{P}$ is greater than $K$, then the enemy population will become extinct, and the plant population will increase to maximum biomass production. When the enemy limits equilibrial plant biomass, then the abundance of the plant and plant enemy can oscillate around the equilibrium. The nature of these oscillations and the local stability of the equilibrium depend on the location of the intersection of the plant and plant enemy zero-growth isoclines (fig. 3). If the isoclines intersect to the right of the peak in the plant isocline $([K-B] / 2>$ $\hat{P})$, the oscillations will dampen over time and the equilibrium will be stable. However, if the isoclines intersect to the left of the plant isocline peak $([K-B] / 2>\hat{P})$, the oscillations will increase over time, and the equilibrium will be locally unstable.

\section{Qualitative Dynamics of the Model}

With this framework, we can make inferences about the expected influence of mycorrhizal fungi on qualitative dynamics by manipulating appropriate parameters. If we assume that the plant enemies depend on the host plant for their entire diet and that plant enemies limit total plant biomass, then simply modifying appropriate parameters associated with each hypothesis suggests possible longterm dynamics of the hypothesis. Under the nutritional quantity hypothesis, mycorrhizal fungi can increase plant access to soil nutrients, thereby increasing the total biomass $(K)$ that plants can sustain. If no other parameters are affected, the plant biomass isocline will increase and the peak $([K-B] / 2)$ will shift to the right (fig. $4 a)$. Surprisingly, when plant mass is limited by the interaction with the enemy, the equilibrium plant population size will not change, while the equilibrium population size of the plant enemy will increase.

In addition to an increase in the carrying capacity, it is also possible that the nutritional quality of the plant may increase through a decrease in defensive chemicals or an increase in nutrient content within plant tissues when associated with mycorrhizal fungi, as described by the nutritional quality hypothesis (fig. $4 b$ ), and this would result in an increase in the efficiency of conversion of plant mass to enemy reproduction $(C)$. This increase in conversion efficiency would cause a shift in the enemy isocline to the left, resulting in a decrease in plant biomass at equilibrium, a probable increase in enemy biomass at equilibrium (depending on the size of the shift and initial starting conditions), and a reduction in the overall stability of the plant-enemy interaction.

If tolerance $(g)$ is increased by mycorrhizal fungi (fig. $4 c$ ), neither the location of the peak of the plant isocline nor the location of the enemy isocline are affected; how- ever, there is change in the overall shape of the plant isocline resulting in an increase in $\hat{E}$. As a result, an association with mycorrhizal fungi leads to an increased equilibrium enemy population size $(\hat{E})$ but no change in the equilibrium plant biomass. We expect no change in the long-term stability of the plant-enemy interaction. Thus under both the nutritional hypotheses and modification of tolerance hypothesis, the improvement in plant nutrition due to mycorrhizal fungi is fully translated into increased population size of the plant enemy and does not result in increased plant population size at equilibrium.

If mycorrhizal fungi lead to an increase in plant defense (fig. $4 d$ ), then the ability of the enemy to convert plant biomass into enemy biomass (the conversion efficiency $C$ ) would decrease because an increase in defensive chemicals might slow herbivore development time. We might also expect an increase in the half-saturation constant $(B)$ because both chemical and mechanical defenses could slow enemy feeding or colonization time. Both a decrease in the conversion efficiency and an increase in the half saturation constant would lead to an increase in equilibrium plant biomass and a decrease in the equilibrium population size of the plant enemy. These changes would shift the enemy isocline to the right, potentially leading to a dampening of oscillations around the equilibrial plant and enemy population size. However, if the plant enemy isocline moves far enough to the right (thus exceeding maximum plant biomass), the enemy population will crash and the plant population will no longer be limited by enemy consumption. Mycorrhizal fungi would probably have qualitatively similar impacts on the interaction between plants and plant enemies under the interference hypothesis because direct interference would also be expected to decrease enemy feeding rate.

\section{Assumptions and Limitations}

As with any generalized model, there are limitations to the theory presented in this article. For example, our discussion assumes that the enemy is able to limit the size of the plant population and that enemy consumption occurs simultaneously with density dependence in the plant population. However, if enemy consumption occurs early in the plant life history and is followed by density-dependent mortality, a decrease in plant biomass could lead to a decrease in intraspecific competition allowing for the possibility that unattacked individuals in the population can make up for the reduced fitness of attacked individuals. Several studies have shown such population-level compensation to enemy attack (Crawley 1988; Crawley 1997; Ditommaso and Watson 1997; Alexander and Mihail 2000). Relaxation of the assumption of simultaneous en- 
a.

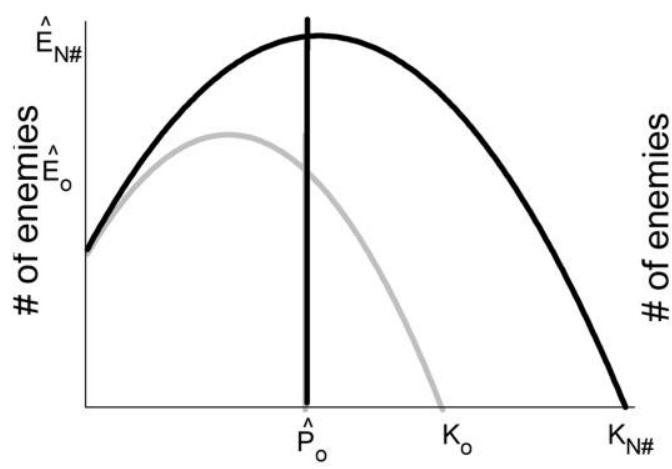

Plant Biomass b.

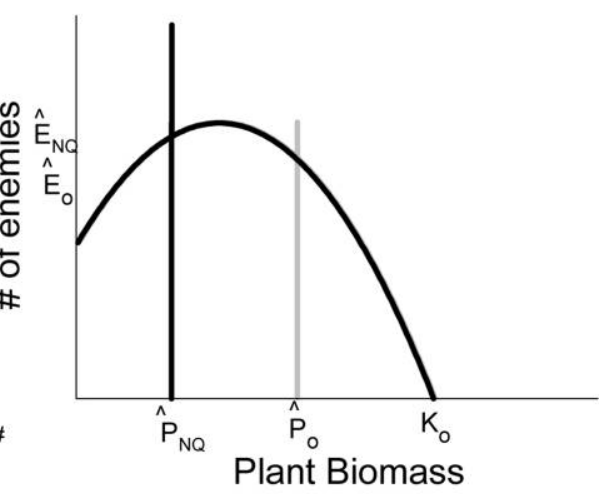

C.

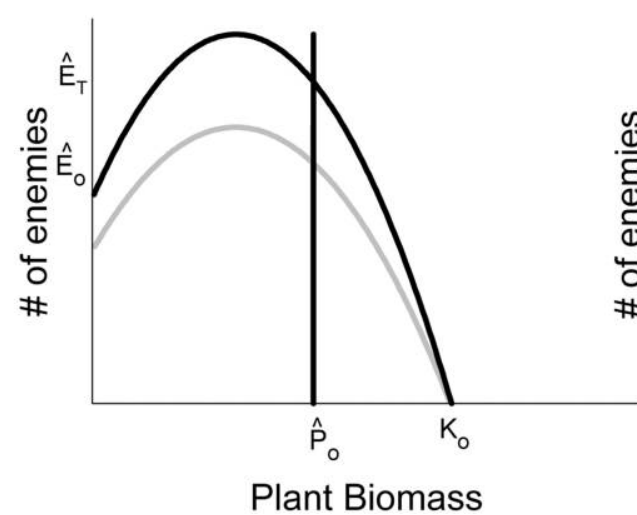

d.

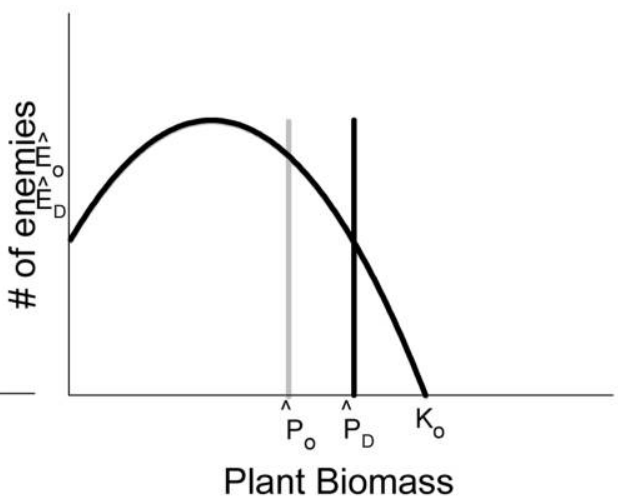

Figure 4: To illustrate the influence of the five hypotheses on plant enemy dynamics, we show the expected alterations from the initial combination of plant and enemy isoclines depicted in figure 3. The initial plant and enemy isoclines from figure 3 are represented as gray lines when they differ from the expected alterations. The original intersection of the plant and enemy isoclines are represented by $\hat{P}_{\mathrm{o}}$ and $\hat{E}_{\mathrm{o}}$. $a$, If the carrying capacity increases, as predicted by the nutritional food quantity hypothesis, then the peak of the plant isocline will shift to the right, but the herbivore isocline will not move. The equilibrium enemy density will increase to $\hat{E}_{\mathrm{N} *}$, but the equilibrium plant density will not change. In this set of isoclines, plant carrying capacity $(K)$ increased from $200\left(K_{\mathrm{o}}\right)$ to $300\left(K_{\mathrm{N} *}\right) . b$, If there is an increase in the efficiency of conversion of plant mass to enemy reproduction $(C)$, as suggested by the nutritional food quality hypothesis, then enemy biomass may increase (if the increase in $C$ is not too great, as denoted by $\hat{E}_{\mathrm{NQ}}$ ), but plant biomass will decrease (as denoted by $\hat{P}_{\mathrm{NQ}}$ ). In our example, $C$ increases from 0.053 to $0.070 . c$, If tolerance of the plant increases, then the equilibrial herbivore density will increase (as denoted by $\hat{E}_{\mathrm{T}}$ ), but the plant biomass will not change. In our example, $t$ increases from 0 to 0.25 . $d$, Alternatively, if there is an increase in herbivore development and feeding rates, as expected with an increase in defense or direct interference, then the enemy isocline will move to the right, which will increase the equilibrium plant density (as denoted by $\hat{P}_{\mathrm{D}}$ ), decrease the equilibrial enemy population size $\left(\hat{E}_{\mathrm{D}}\right)$, and increase the stability of the interaction. In our example, $C$ decreases from 0.053 to 0.05 .

emy consumption and density dependence could alter the predicted dynamics.

We assume that the plant enemy solely consumes the target plant population. This will be the case for specialist plant enemies but is unlikely to hold true for complete generalists such as ungulates. Rather, the equilibrium densities of the ungulates will probably be a function of the entire plant community rather than a particular plant population. We therefore focus our discussion on herbivores and plant pathogens whose densities are more likely to be responsive to changes in mass of a given plant population.
We also assume that the enemy population is limited by the availability of this resource. If enemy population size is limited by higher trophic levels, then the predicted equilibrium population sizes could be strongly affected. The potential impacts of mycorrhizal fungi on these higher trophic-level interactions is an interesting subject of investigation (Gange et al. 2003; Guerrieri et al. 2004). Research has suggested that an association with mycorrhizal fungi might alter parasitoid behavior through changes in plant size (that affect search efficiency; Gange et al. 2003) or changes in the release of volatiles attractive to parasit- 
oids (Guerrieri et al. 2004). Simple alterations of parasitoid behavior, for example, are likely to change the simple predicted outcome between plants and plant enemies examined in this model.

In this theory, we have assumed that mycorrhizal fungi are beneficial to plant growth. The net benefits of mycorrhizal fungi to plants, however, depends on many abiotic and biotic factors. When the costs of the fungi outweigh the benefits, the fungi will be pathogenic. We note however that such pathogenic fungi could alter plant mass, food quality, tolerance to herbivory, and plant defenses. Therefore, the general approach taken here could also be used to investigate the qualitative dynamic of plant-pathogen-herbivore interactions.

\section{Discussion}

The theory developed in this article shows how potential mechanisms for mycorrhizal effects on plant growth, tolerance, or enemy defense can have qualitatively different influences on long-term dynamics between plants and plant enemies (see above). Under the nutritional quality, nutritional quantity, and modification of tolerance hypotheses, despite the potential for increases in growth or nutritional quality of individual plants, mycorrhizal fungi are not expected to increase plant biomass at equilibrium. Instead, the higher plant growth conferred by mycorrhizal fungi results in equilibrial increases in enemy biomass. In contrast, under the modification of defense and interference hypotheses, association with mycorrhizal fungi causes plant biomass at equilibrium to increase while enemy biomass at equilibrium decreases. There is evidence for the assumptions and predictions of each of these mechanisms (see table A1 in the online edition of the American Naturalist). However, at present, we do not have enough information to generalize about the conditions in which we are likely to see these different mechanisms. First, it is possible that multiple mechanisms may be acting at once, but there are also a number of factors that might shift a plant response, and thus potential enemy population growth rates, such as type of herbivore, type of mycorrhizal symbiont, plant successional status, and abiotic factors, to name a few.

\section{Multiple Mechanisms of Plant Response}

Mycorrhizal fungi can affect the interactions between plants and plant enemies in multiple ways simultaneously. While a trade-off in resource allocation can constrain the magnitude of plant response, mycorrhizal fungi could both increase plant mass (and therefore available food) and decrease food quality or increase allocation to both growth and storage (combining effects of increased growth rates and tolerance). It is also possible that mycorrhizal fungi could increase plant defenses against root pathogens while simultaneously directly interfering with root infection by that pathogen. For example, in a split root experiment conducted by Pozo et al. (2002), pathogens in the nonmycorrhizal half of pots still experienced a decrease in fitness despite the lack of mycorrhizal fungi occupying root space. It is also possible that mycorrhizal fungi will alter the interaction between a plant and one enemy in one way and the interaction between the same plant and a different enemy in a qualitatively different way.

\section{Type of Plant Enemy}

Varied plant responses to a variety of enemies seem particularly likely given that plant enemies differ in their susceptibility to plant defenses. Parasites and specialist herbivores, for example, are probably able to circumvent the defenses of their host plant and remain undetected (van der Meijden 1996; Manninen 1999). As a result, these enemies would likely benefit from a host plant's association with mycorrhizal fungi even if it does result in an increase in secondary chemicals. Alan Gange and collaborators (Gange et al. 2002a) surveyed the flora of the United Kingdom and found a positive correlation between mycorrhizal status and the number of specialist herbivores feeding on that plant family. Indeed, specialist herbivores have often been shown to increase in fitness due to an association with mycorrhizal fungi in a number of studies (Rabin and Pacovsky 1985; Sanders et al. 1993; Gange and West 1994; Borowicz 1997; Gange et al. 2002a). Parasitic plants might also be expected to benefit by feeding on hosts with a mycorrhizal association because they often directly tap into the xylem or phloem of a host plant, thereby avoiding plant defenses. Only one study has examined aboveground plant parasites, and it found that mycorrhizal fungi did increase the biomass of the parasitic plant Dodder (Sanders et al. 1993). Generalist herbivores may be more susceptible to plant secondary chemicals (van der Meijden 1996; Manninen 1999) and therefore may be more negatively impacted by the same plant's association with mycorrhizal fungi, although this has never been directly tested. Gange et al. (2002a) tested for the dichotomy between specialist and generalist herbivores in three species of Lamiaceae and found that specialist herbivores in his system were more positively affected by association with mycorrhizal fungi than were generalist herbivores on these same hosts. Root pathogens also tend to respond in a similar manner to generalist herbivores in the presence of mycorrhizae. In a meta-analysis, Borowicz (2001) showed that, depending on the timing of infection, mycorrhizae tend to decrease pathogen growth rates. Thus, even if an aphid and a pathogen attack at the same moment in time, 
they may be affected in qualitatively different manners by the plant's association with mycorrhizal fungi.

\section{Type of Mycorrhizal Fungi}

There are several types of mycorrhizal fungi, all of which vary in the type and ratio of soil nutrients they access. All of the research on multitrophic interactions involving mycorrhizal fungi has focused on two of these types: arbuscular mycorrhizal fungi and ectomycorrhizal fungi. Ectomycorrhizae are considered more important in nitrogen uptake, and thus they might prove more important for plant defenses that contain nitrogen or require nitrogen for synthesis. Arbuscular mycorrhizal fungi play a greater role in phosphorous uptake, and thus they might be thought to contribute to plant nutrition more than defense because phosphorous is not considered as essential for defense or synthesis of defensive compounds. However, at present the literature (as cited above) does not reveal a definite pattern between the two types of fungi. As a result, more research is needed to determine whether a pattern between fungal types exists.

\section{Plant Successional Status}

Our model predicts that plant allocation of the benefits derived from mycorrhizal fungi to growth or tolerance will lead to greater enemy fitness when enemy population is low, but not when the enemy reaches equilibrium population size. Conversely, plant allocation of the benefits derived from mycorrhizal fungi to defense may not lead to greater plant fitness when enemy population size is low but will lead to a greater realized fitness as enemy populations approach their equilibrium levels. From these predicted trade-offs in the two allocation strategies, we can expect a correlation between the plant allocation of mycorrhizal benefits and plant life history. In particular, short-lived, early-successional species that rapidly colonize areas after disturbance only to be subsequently excluded by stronger competitors may be unlikely to experience equilibrium densities of herbivores and would maximize their fitness by allocation of the benefits from mycorrhizal fungi to increased growth (either increasing $K$ or $t$ ) rather than defense. On the other hand, long-lived and latesuccessional plant species remain in one place for long periods of time and therefore cannot escape the effects of plant enemies by dispersing to new locations. As a result they would be more likely to live through multiple enemy generations and experience equilibrium. Thus, we might expect that long-lived and late-successional species would maximize their fitness by allocation of the benefits derived from mycorrhizal fungi to increased defense (increasing $B$ and/or $C$ ).
If these predictions hold true, then we ought to see more annuals falling within the nutritional and tolerance hypotheses and more perennials falling within the modification of defense hypothesis. A cursory examination of the literature provides support for these predictions. In all of the annual species examined-Plantago lanceolata (Gange et al. 1999, 2002b), soybean (Borowicz 1997), and Abutilon theophrasti (Sanders et al. 1993) - herbivore fitness increased on host plants associated with mycorrhizal fungi as predicted by the nutritional hypotheses. However, additional studies with two of these species-Plantago lanceolata (Gange and West 1994) and soybean (Rabin and Pacovsky 1985)—demonstrated a decline in herbivore fitness on host plants associated with mycorrhizal fungi, suggesting a need for further study. Studies found that in five out of nine perennial species examined-cottonwood (Gehring and Whitham 2002), Scots pine (Manninen et al. 1998, 1999, 2000), Cirsium arvense (Gange and Nice 1997), oak (Rieske 2001), and perennial rye grass (Vicari et al. 2002) — herbivore fitness declined on host plants associated with mycorrhizal fungi. However, in two out of five of these species-cottonwood (Gehring and Whitham 2002) and Scots pine (Manninen et al. 1999, 2000)—different herbivores also saw an increase in fitness when feeding on mycorrhizal hosts. While these patterns are consistent with the expectations, more studies are needed before the predictions can be properly tested.

\section{Abiotic Factors}

Abiotic factors play a role in many interactions, and so it would not be surprising that abiotic factors such as water limitation or nutrient gradients might alter the outcome of multitrophic interactions involving mycorrhizae, plants, and plant enemies. Two studies involving arbuscular mycorrhizal fungi have included fertility gradients of phosphorous, and both showed that defense-related responses decreased in plants associated with mycorrhizal fungi as soil phosphorous concentrations increased (Borowicz 1997; Gange et al. 1999). Both studies suggest that environmental factors are likely to play a role in how plants respond to plant enemies in the presence of mycorrhizal fungi and that more studies examining nutrient gradients and other abiotic factors in the context of multitrophic interactions are needed.

\section{Conclusion}

One goal of ecological research is to dissect interactions within communities in order to predict when those interactions might change. Complex communities are difficult to break down, so we often approach them from the simplest perspective possible: pairwise interactions. In 
some systems, simply combining the separate effects of mycorrhizae, plants, and plant enemies will be adequate where mycorrhizal effects are mediated primarily by plant mass (as in the case of the nutritional quality hypothesis). All of the other mechanisms proposed in this article suggest that a simple combination of pairwise interactions would fail to predict the outcome of multitrophic interactions involving mycorrhizae, plants, and herbivores.

The hypotheses identified in this article contrast qualitatively different ways in which mutualistic association with mycorrhizal fungi can affect antagonistic interactions between plants and plant enemies. In addition, the theory we developed provides a qualitative prediction of the longterm implications of the different mechanisms we identify. Perhaps most surprisingly, we find that in the absence of an increased allocation to defense, an increase in nutritional resources due to association with mycorrhizal fungi will not increase plant population size at equilibrium when plants are regulated by plant enemies. We find evidence supporting the operation of most of the mechanisms in particular systems, but much work remains in identifying the patterns in which we are likely to encounter these mechanisms.

\section{Acknowledgments}

We would like to thank the following people for taking the time to review and discuss the article with us: E. Allen, M. Allen, M. Damm, J. Kang, W. Konoungbua, T. Paine, H. Reynolds lab group, S. Richardson, J. Rudgers, P. Schultz, J. Stevens, W. Taheri, K. Vogelsang, and S. Wooley. This article would not be the same without their help! In addition we thank R. Karban and J. Klironomos for helpful suggestions on the manuscript. We also acknowledge the support of National Science Foundation grants DEB0407816 to A.E.B. and DEB-0049080 to J.D.B.

\section{Literature Cited}

Alexander, H. M., and J. D. Mihail. 2000. Seedling disease in an annual legume: consequences for seedling mortality, plant size, and population seed production. Oecologia (Berlin) 122:346-353.

Baldwin, I. T. 1994. Chemical changes rapidly induced by folivory. Pages 1-23 in E. A. Bernays, ed. Insect-plant interactions. CRC, Boca Raton, FL.

Bever, J. D. 2002. Negative feedback within a mutualism: host-specific growth of mycorrhizal fungi reduces plant benefit. Proceedings of the Royal Society of London B 269:2595-2601.

Borowicz, V. A. 1997. A fungal root symbiont modifies plant resistance to an insect herbivore. Oecologia (Berlin) 112:534-542.

- 2001. Do arbuscular mycorrhizal fungi alter plant-pathogen relations? Ecology 82:3057-3068.

Caughley, G. 1976. Plant-herbivore systems. Pages 94-113 in R. M. May, ed. Theoretical ecology: principles and applications. Blackwell Scientific, Oxford.

Charron, G., V. Furlan, M. Bernier-Cardou, and G. Doyon. 2001.
Response of onion plants to arbuscular mycorrhizae. 1. Effects of inoculation method and phosphorus fertilization on biomass and bulb firmness. Mycorrhiza 11:187-197.

Coley, P. D., J. P. Bryant, and F. S. Chapin. 1985. Resource availability and plant antiherbivore defense. Science 230:895-899.

Crawley, M. J. 1983. Herbivory: the dynamics of animal-plant interactions. Studies in Ecology. Vol. 10. Blackwell Scientific, Oxford.

. 1988. Herbivores and plant population dynamics. Pages 367392 in A. J. Davy, M. J. Hutchings, and A. R. Watkinson, eds. Plant population ecology. 28th British Ecological Society Symposium. Blackwell Scientific, Oxford.

. 1997. Plant-herbivore dynamics. Pages 401-474 in M. J. Crawley, ed. Plant ecology. Blackwell Science, Cambridge.

Ditommaso, A., and A. K. Watson. 1997. Effect of the fungal pathogen, Colletotrichum coccodes, on Abutilon theophrasti height hierarchy development. Journal of Applied Ecology 34:518-529.

Edelstein-Keshet, L. 1988. Mathematical models in biology. Random House, New York.

Fineblum, W. L., and M. D. Rausher. 1995. Tradeoff between resistance and tolerance to herbivore damage in a morning glory. Nature 377:517-520.

Fornoni, J., P. L. Valverde, and J. Nunez-Farfan. 2003. Quantitative genetics of plant tolerance and resistance against natural enemies of two natural populations of Datura stramonium. Evolutionary Ecology Research 5:1049-1065.

Fusconi, A., E. Gnavi, A. Trotta, and G. Berta. 1999. Apical meristems of tomato roots and their modifications induced by arbuscular mycorrhizal and soilborne pathogenic fungi. New Phytologist 142: 505-516.

Gange, A. C., and V. K. Brown. 2002. Actions and interactions of soil invertebrates and arbuscular mycorrhizal fungi in affecting the structure of plant communities. Pages 321-344 in M. G. A. van der Heijden and I. R. Sanders, eds. Mycorrhizal ecology. Ecological Studies. Vol. 157. Springer, Berlin.

Gange, A. C., and H. E. Nice. 1997. Performance of the thistle gall fly, Urophora cardui, in relation to host plant nitrogen and mycorrhizal colonization. New Phytologist 137:335-343.

Gange, A. C., and H. M. West. 1994. Interactions between arbuscular mychorrhizal fungi and foliar-feeding insects in Plantago lanceolata L. New Phytologist 128:79-87.

Gange, A. C., E. Bower, and V. K. Brown. 1999. Positive effects of an arbuscular mycorrhizal fungus on aphid life history traits. Oecologia (Berlin) 120:123-131.

Gange, A. C., P. G. Stagg, and L. K. Ward. 2002a. Arbuscular mycorrhizal fungi affect phytophagous insect specialism. Ecology Letters 5:11-15.

Gange, A. C., E. Bower, and V. K. Brown. 2002b. Differential effects of insect herbivory on arbuscular mycorrhizal colonization. Oecologia (Berlin) 131:103-112.

Gange, A. C., V. K. Brown, and D. M. Aplin. 2003. Multitrophic links between arbuscular mycorrhizal fungi and insect parasitoids. Ecology Letters 6:1051-1055.

2005. Ecological specificity of arbuscular mycorrhizae: evidence from foliar- and seed-feeding insects. Ecology 86:603-611.

Gehring, C. A., and T. G. Whitham. 2002. Mycorrhizae-herbivore interactions: population and community consequences. Pages 295320 in M. G. A. van der Heijden and I. R. Sanders, eds. Mycorrhizal ecology. Ecological Studies. Vol. 157. Springer, Berlin.

Gehring, C. A., N. S. Cobb, and T. G. Whitham. 1997. Three-way interactions among ectomycorrhizal mutualists, scale insects, and 
resistant and susceptible pinyon pines. American Naturalist 149: 824-841.

Goverde, M., M. G. A. van der Heijden, A. Wiemken, I. R. Sanders, and A. Erhardt. 2000. Arbuscular mycorrhizal fungi influence life history traits of a lepidopteran herbivore. Oecologia (Berlin) 125: 362-369.

Guerrieri, E., G. Lingua, M. C. Digilio, N. Massa, and G. Berta. 2004. Do interactions between plant roots and the rhizosphere affect parasitoid behaviour? Ecological Entomology 29:753-756.

Harrison, G. W. 1995. Comparing predator-prey models to Luckinbill's experiment with didinium and paramecium. Ecology 76: $357-374$.

Johnson, N. C., J. H. Graham, and F. A. Smith. 1997. Functioning of mycorrhizal associations along the mutualism-parasitism continuum. New Phytologist 135:575-586.

Karban, R., and I. T. Baldwin. 1997. Induced responses to herbivory: interspecific interactions. University of Chicago Press, Chicago.

Klironomos, J. N. 2003. Variation in plant response to native and exotic arbuscular mycorrhizal fungi. Ecology 84:2292-2301.

Kudo, G. 1996. Herbivory pattern and induced responses to simulated herbivory in Quercus mongolica var. grosseserrata. Ecological Research 11:283-289.

Kula, A. A. R., D. C. Hartnett, and G. W. T. Wilson. 2005. Effects of mycorrhizal symbiosis on tallgrass prairie plant-herbivore interactions. Ecology Letters 8:61-69.

Loomis, W. E. 1932. Growth-differentiation balance versus carbohydrate-nitrogen ratio. Proceedings of the American Society for Horticultural Science 29:240-245.

-1953. Growth and differentiation: an introduction and summary. Pages 1-17 in W. E. Loomis, ed. Growth and differentiation in plants. Iowa State College Press, Ames.

Manninen, A.-M. 1999. Susceptibility of Scots pine seedlings to specialist and generalist insect herbivores: importance of plant defense and mycorrhizal status. PhD thesis. University of Kuopio, Finland.

Manninen, A.-M., M. Vuorinen, and J. K. Holopainen. 1998. Variation in growth, chemical defense, and herbivore resistance in Scots pine provenances. Journal of Chemical Ecology 24:1315-1331.

Manninen, A.-M., T. Holopainen, and J. K. Holopainen. 1999. Performance of grey pine aphid, Schizolachnus pineti, on ectomycorrhizal and non-mycorrhizal Scots pine seedlings at different levels of nitrogen availability. Entomologia Experimentalis et Applicata 93:117-120.

Manninen, A.-M., T. Holopainen, P. Lyytikainen-Saarenmaa, and J. K. Holopainen. 2000. The role of low-level ozone exposure and mycorrhizas in chemical quality and insect herbivore performance on Scots pine seedlings. Global Change Biology 6:111-121.

McAllister, C. B., I. Garcia-Romera, J. Martin, A. Godeas, and J. A. Ocampo. 1995. Interaction between Aspergillus niger van Tiegh. and Glomus mosseae (Nicol. \& Gerd.) Gerd. and Trappe. New Phytologist 129:309-316.

McPeek, M. A., and T. E. Miller. 1996. Evolutionary biology and community ecology. Ecology 77:1319-1320.

Miller, R. M., S. P. Miller, J. D. Jastrow, and C. B. Rivetta. 2002. Mycorrhizal mediated feedbacks influence net carbon gain and nutrient uptake in Andropogon gerardii. New Phytologist 155:149162.

Miller, T. E., and J. Travis. 1996. The evolutionary role of indirect effects in communities. Ecology 77:1329-1335.

Mole, S. 1994. Trade-offs and constraints in plant-herbivore defense theory: a life-history perspective. Oikos 71:3-12.
Newsham, K. K., A. H. Fitter, and A. R. Watkinson. 1995. Multifunctionality and biodiversity in arbuscular mycorrhizas. Trends in Ecology \& Evolution 10:407-411.

Paige, K. N., and T. G. Whitham. 1987. Overcompensation in response to mammalian herbivory: the advantage of being eaten. American Naturalist 129:407-416.

Palermo, B. L., K. M. Clancy, and G. W. Koch. 2003. The potential role of ectomycorrhizal fungi in determining Douglas-fir resistance to defoliation by the western spruce budworm (Lepidoptera: Tortricidae). Journal of Economic Entomology 96:783-791.

Pozo, M. J., C. Cordier, E. Dumas-Gaudot, S. Gianinazzi, J. M. Barea, and C. Azcon-Aguilar. 2002. Localized versus systemic effect of arbuscular mycorrhizal fungi on defence responses to Phytophthora infection in tomato plants. Journal of Experimental Botany 53: 525-534.

Prittinen, K., J. Pusenius, K. Koivunoro, and H. Roininen. 2003. Genotypic variation in growth and resistance to insect herbivory in silver birch (Betula pendula) seedlings. Oecologia (Berlin) 137: 572-577.

Rabin, L. B., and R. S. Pacovsky. 1985. Reduced larva growth of two lepidoptera (Noctuidae) on excised leaves of soybean infected with a mycorrhizal fungus. Lournal of Economic Entomology 78:13581363.

Rhoades, D. F. 1979. Evolution of plant chemical defense against herbivores. Pages 1-55 in G. A. Rosenthal and D. H. Janzen, eds. Herbivores: their interaction with secondary plant metabolites. Plenum, New York.

Rieske, L. K. 2001. Influence of symbiotic fungal colonization on oak seedling growth and suitability for insect herbivory. Environmental Entomology 30:849-854.

Rieske, L. K., C. C. Rhoades, and S. P. Miller. 2003. Foliar chemistry and gypsy moth, Lymantria dispar (L.), herbivory on pure American chestnut, Castanea dentata (Fam: Fagaceae), and a diseaseresistant hybrid. Environmental Entomology 32:359-365.

Rosenzweig, M. L., and R. H. MacArthur. 1963. Graphical representation and stability conditions of predator-prey interactions. American Naturalist 97:209.

Sanders, I. R., R. T. Koide, and D. L. Shumway. 1993. Mycorrhizal stimulation of plant parasitism. Canadian Journal of Botany 71: 1143-1146.

Sen, R. 2001. Multitrophic interactions between a Rhizoctonia sp. and mycorrhizal fungi affect Scots pine seedling performance in nursery soil. New Phytologist 152:543-553.

Slezack, S., E. Dumas-Gaudot, S. Rosendahl, R. Kjoller, M. Paynot, J. Negrel, and S. Gianinazzi. 1999. Endoproteolytic activities in pea roots inoculated with the arbuscular mycorrhizal fungus Glomus mosseae and/or Aphanomyces euteiches in relation to bioprotection. New Phytologist 142:517-529.

Smith, S. E., and D. J. Read. 1997. Mycorrhizal symbiosis. Academic Press, San Diego, CA.

Stowe, K. A. 1998. Experimental evolution of resistance in Brassica rapa: correlated response of tolerance in lines selected for glucosinolate content. Evolution 52:703-712.

Strauss, S. Y., and A. A. Agrawal. 1999. The ecology and evolution of plant tolerance to herbivory. Trends in Ecology \& Evolution 14: 179-185.

Strauss, S. Y., W. Watson, and M. T. Allen. 2003. Predictors of male and female tolerance to insect herbivory in Raphanus raphanistrum. Ecology 84:2074-2082.

Strong, D. R., J. H. Lawton, and R. Southwood. 1984. Insects on 
plants: community patterns and mechanisms. Harvard University Press, Cambridge, MA.

Sundaresan, P., N. U. Raja, and P. Gunasekaran. 1993. Induction and accumulation of phytoalexins in cowpea roots infected with a mycorrhizal fungus glomus-fasciculatum and their resistance to Fusarium-Wilt disease. Iournal of Biosciences 18:291-301.

van de Koppel, J., J. Huisman, R. van der Wal, and H. Olff. 1996. Patterns of herbivory along a productivity gradient: an empirical and theoretical investigation. Ecology 77:736-745.

van der Heijden, M. G. A., T. Boller, A. Wiemken, and I. R. Sanders. 1998. Different arbuscular mycorrhizal fungal species are potential determinants of plant community structure. Ecology 79:20822091.

van der Meijden, E. 1996. Plant defence, an evolutionary dilemma: contrasting effects of (specialist and generalist) herbivores and natural enemies. Entomologia Experimentalis et Applicata 80:307310.

Vicari, M., P. E. Hatcher, and P. G. Ayres. 2002. Combined effect of foliar and mycorrhizal endophytes on an insect herbivore. Ecology 83:2452-2464.

Vos, M., B. Kooi, D. DeAngelis, and W. Mooij. 2004. Inducible defences and the paradox of enrichment. Oikos 105:471-480.

Wootton, J. T. 1994. Predicting direct and indirect effects: an integrated approach using experiments and path analysis. Ecology 75: $151-165$.

Wurst, S., D. Dugassa-Gobena, R. Langel, M. Bonkowski, and S. Scheu. 2004. Combined effects of earthworms and vesiculararbuscular mycorrhizas on plant and aphid performance. New Phytologist 163:169-176.

Yodzis, P. 1989. Introduction to theoretical ecology. Harper \& Row, New York.

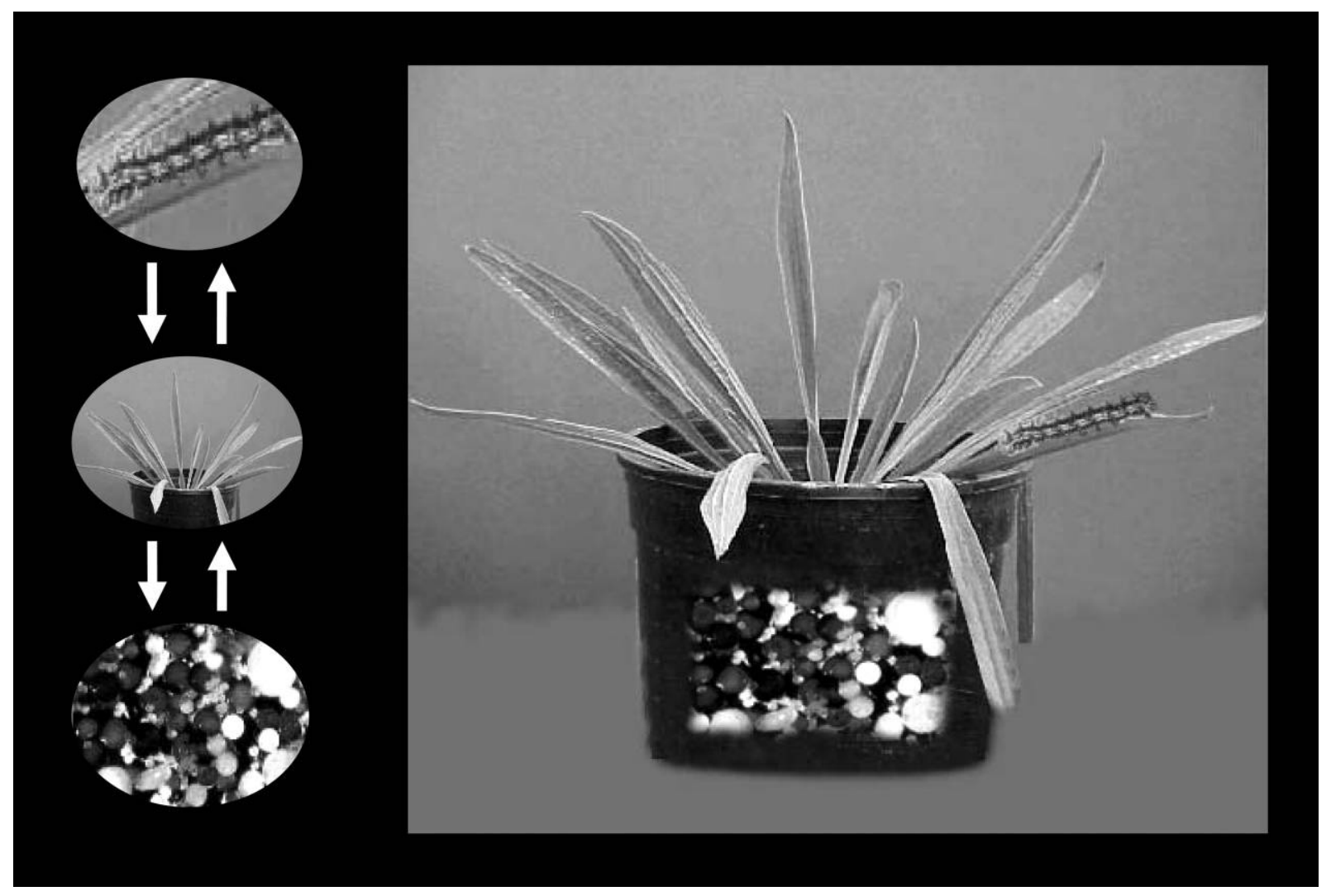

An example of interactions between mycorrhizae, plants, and plant enemies. 